\title{
Dunhuang Caisson Patterns in Fashion Design
}

\author{
Yumin CAO, Jing WANG, Huaming PENG* \\ South China University of Technology
}

\begin{abstract}
This study analyzed the current status quo of Dunhuang caisson pattern, extracted and redesigned its constituent elements. By researching through scattering the constituent elements of pattern, deconstruction and re-integration, extracting and stitching the color, and combined with aesthetic styles of modern design, we can apply new design patterns to fashion design.
\end{abstract}

KEYWORD: Dunhuang caisson pattern; Modern design aesthetic style; Fashion design

The Mogao Grottoes in Dunhuang is located in the western end of the Gansu Corridor, it is famous by fresco and statue. The patterns came from four Buddha lotus of the 314 Mogao Grottoes of China Art Gallery were discussed in this paper. The Mogao Grottoes is the pearl of the silk road in China, and the silk road is the cultural tie that connects India and China. As the communications centre, the Mogao Grottoes represents the spread of Buddhist art as well as foreign culture in China. Four Buddha lotus pattern is come from the murals building of the Mogao Grottoes, with the introduction of Buddhism, it is widely used in building decoration in the Sui Dynasty, lotus is the symbol of purity, noble and good luck in Buddhism.

Cao Zhi once wrote in his famous article "Lotus Fu", "look at the beauty of all the flowers, you can merely find something else as elegant and spiritual like that ". "In Kwan Fong spectrum", "All things first show gorgeous and then the real, only this gorgeous is inherent. Rising is connected and communicated with a smart, beautiful, graceful posture, come out of mud, but not dyed, gentleman of the flower". "As a precious cultural heritage of mankind, the Mogao Grottoes has a high value of researching. Through the study of the Mogao Grottoes murals, we can get further understanding of graphic design of the traditional idea, to analyze the traditional cultural significance of patterns, and to analyze the manifestation of the pattern in modern

1 The cultural significance of lotus in Wikipedia, explain in Chinese culture, "the ancient Chinese writer think lotus mean holy and elegant,so in ancient poetry,there is chapter praised lotus." design, finally, to explore its application in fashion design, it has a certain degree of motivating value for the promotion of Chinese traditional culture and modern art.

\section{RESEARCH STATUS QUO OF DUNHUANG CAISSON PATTERNS}

Dunhuang caisson patterns are widely used in decorative design in modern applications, usually researched by the composed of Dunhuang caisson structure, color patterns, organizational relationships in the pattern, the elements extraction of the pattern and other aspects study. "The simplicity in complex - analytic Dunhuang caisson pattern" (Chen Xiongjun, Liang Zhaohua), through researching the characteristics of "complicated but not chaotic" of Dunhuang caisson patterns, to explore the modeling laws of Dunhuang caisson pattern from tedious to simplicity, mainly reflected in structure by the grid of the basic structure of the Chinese character "mi", and the use of the hierarchical organization skills[1].
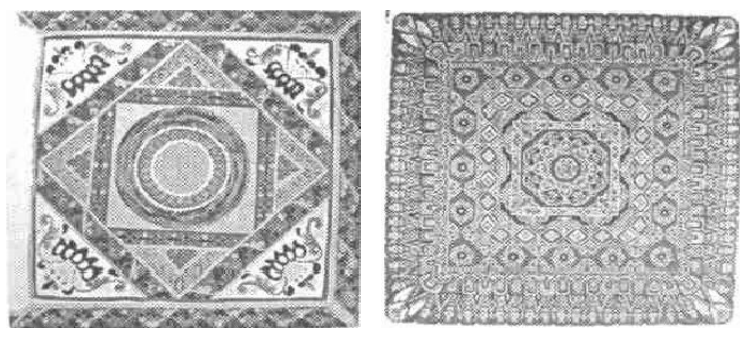

Figure 1. The map of Lotus Honeysuckle caisson Figure 2. Floral caisson 

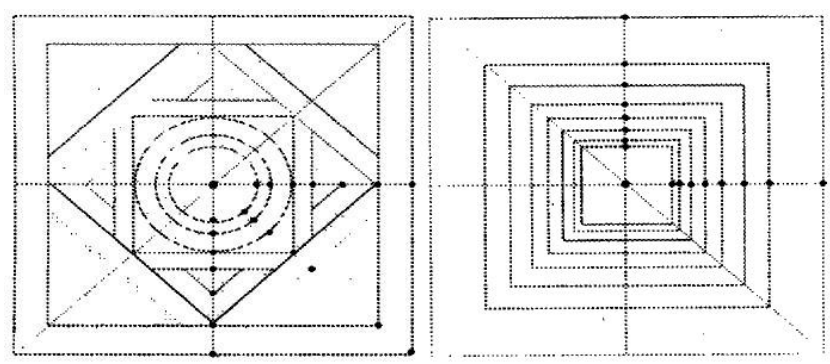

Figure 3.The diagram of Lotus Honeysuckle caisson

Figure 4.The structure diagram of Floral caisson

Pattern Source: "The numerous simplify - analytic Dunhuang caisson pattern" (Chenxiong Jun, Liang Zhaohua)

Figure 3 shows the Lotus honeysuckle caisson structure diagram of the Mogao in Western Wei Dynasty, Figure 4 shows the structure diagram of Flora caisson in Tang Dynasty. Both of the forms are of the basic structure of the Chinese character "mi". In the middle is the square well, so that it is beneficial to keep equilibrium and stability in terms of the intensity of different directions. The form of Caisson is changed by the center of the word lattice, the diagonal points distance is used to determine the distance of the width of the square, the "mi" word lattice framework have stable characteristics, widely used in the Tang Dynasty murals.

"Dunhuang caisson pattern in modern decor Design" (Liu Tao, Peng Mai Fu), Analysis of the use of Dunhuang caisson pattern in the design of the Great Hall of the People, that is the early example of its application in architectural design [2].
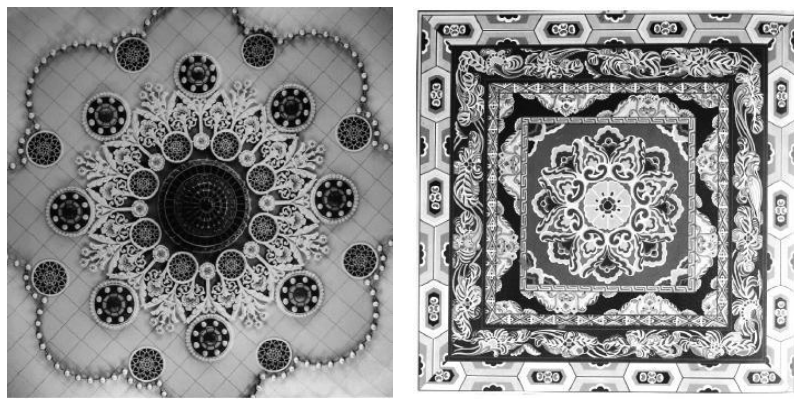

Figure 5. The caisson pattern of Dunhuang Mogao Cave 31 Figure 6. The patio design of Great Hall Ballroom (Chang Shana)

Pattern Source: "Dunhuang caisson pattern in modern interior design application," Liu Tao, Peng Maifu

At the International Dunhuang frescoes Inheritance and Innovation Symposium, 2007. Mr. Chang Shana pointed the element of design in Great Hall ballroom ceiling similar to the caisson Lotus of Tang Mogao Cave 31 (Figure6), the design style from the Tang Dynasty caisson decoration, flower relief in the form of gypsum, combine functionality requires lighting, ventilation, building structure, consist of the unique national style of the City Hall.

"On the Art of Color Dunhuang murals and caisson pattern" (Yang Guixiang College of Qinghai
Teachers, 2006), she described the application of color patterns on the caisson, before the Sui Dynasty, color application based with large blocks, simple color, the main techniques for blooming color to red ocher soil and decorated with green and white foil, black hook line, and then match the shape of the coarse ore bold. More bright and lively tone applied at Sui, then, from Sui to Tang Dynasty, appeared azurite, cinnabar, ink, stone yellow, indigo and other colors, color became more abundant ."On the modern dyeing patterns from Dunhuang caisson design pattern" (Xiao Xiao, Art Space), She proposed the method of how to in-depth reference pattern Dunhuang caisson, refined and extended the application of the caisson Grids," Fang Well - trim vertical mantle "three-tier architecture and modern patterns constitute symmetry, balance, the principle of rhythm, rhythm, change, unity, specificity, consistent space. Refine and regeneration in two ways, $\mathrm{A}$ is extraction and generation and $\mathrm{B}$ is deconstruction and integration[3]. Put forward two ways in color elements made caisson refinement and extension: put the typical color of Dunhuang in modern design directly, or use the caisson color performance practices rhythm and color simply.

In recent years, the caisson pattern has apply in design of Scarf and scarves, such as the "Prince Gong's Mansion," "Beijing impression", caisson structure of the word "mi" lattice pattern used in scarf designs, this well-organized structure fill with a representative element of Beijing, such as building patterns, silhouette patterns, which designed with a modern style all. In addition, in the exhibition of WHO'S NEXT at 2013, the brand of Hess -Sunfed was invited as China original designer of high-end brands, "Dunhuang" and "robe" series were the highlight in its fashion design. series of "Dunhuang" from the Mogao Grottoes in Korea caisson fan pattern evolved out of silk fabric with stitching and modern tailoring, stimulate female vitality ${ }^{2}$.

\section{RESEARCH METHODS OF CAISSON PATTERNS APPLIED IN THE CLOTHING}

Collect and research the current related disciplines of literature, it can be seen most Dunhuang caisson pattern starting grid structure word "mi" from the structure, look for an entry point in the square; the study of the pattern of color multi-start its inherent color, the natural color apply directly to design or the painting techniques apply to design. In addition to the inherent color in color combination of modern applications; Extract and apply the element which on behalf of the central idea in the pattern, or apply the element after its restructuring. Qualitative analysis

\footnotetext{
2 Oriental Financial. Zhou Hao at 8:55 on January 21, 2013
} 
method, study with the 314 of Mogao Grottoes caisson pattern, summarize and analysis the method of literature studies from the pattern above for design.

\section{APPLICATION OF DUNHUANG CAISSON PATTERNS IN FASHION DESIGN}

\subsection{Extract and apply the elements of Dunhuang caisson pattern}

Lotus pattern is the main element of the center of four Buddha picture, by extracting patterns of lotus, then simplification, segmentation, extracting part of the elements, extend the elements to four directions continuously for get new graphics.

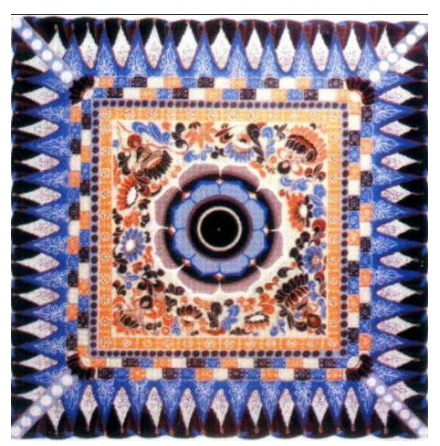

Pattern Source: China Art Gallery 314 Mogao Grottoes four Buddha lotus (Sui)
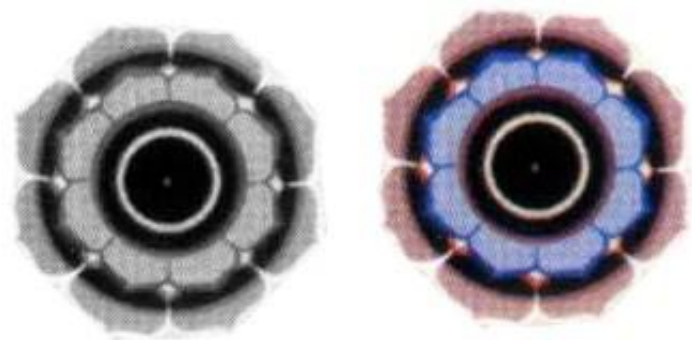

Figure 7. Extract elements of Lotus

Figure 8. PS colorless processing elements of Lotus

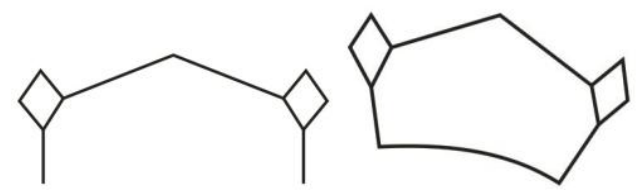

Figure 9. Extract individual constituent elements

Figure 10. New element after deformation and simplify

Repeat and extend the elements to four directions continuously to form a new form of pattern.
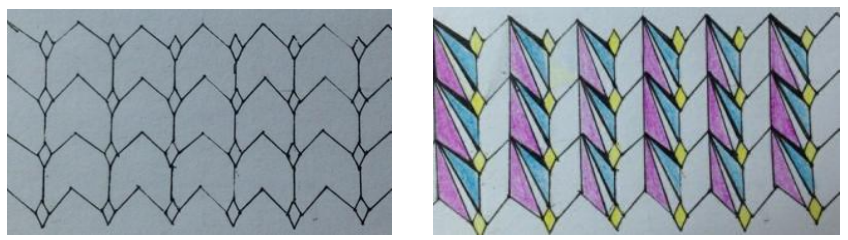

Figure 11. Pattern of quartet continuous

Figure 12. Pattern (a)
Using other methods to form individual segmentation; enhanced color contrast, convert the original primary color for higher brightness color to obtain a new pattern (a); Obtaining original pattern color of lotus and the color from figure six to unify pattern (b), then combined the pattern (a) and pattern (b) retain the meaning and value of the original lotus pattern, combined with other forms of graphics with the application of the split in costume design.
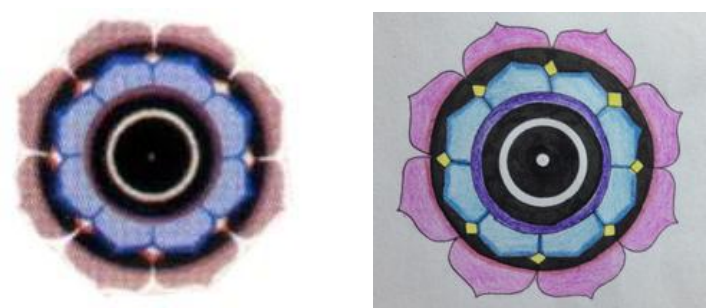

Figure 13. Pattern (b)

In Figure14, application of the lotus mainly in the skirt, adopted triangle composition, broke up the color of Figure 12 for use to achieve balanced results. The main tool for drawing are color pencils, use Marley paint wood color of lead in 05 yellow, 07 light blue, 11 purple, 14 red roses with black and white. Works retain pure and elegant implication of the original four Buddha lotus, add modern art division. In today's society, most young women want to dress with personality characteristics, distinctive, and be able to show their own advantages. Not only embodies the traditional Chinese mural culture, but also show the modern sense of fashion design method.

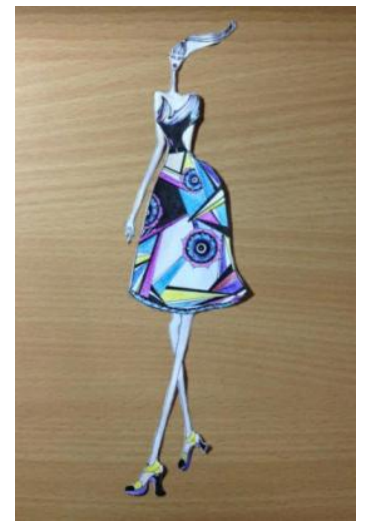

Fig.14 Pattern sketch of dress (a)

\subsection{Decomposed, broken up, regrouped the} constituent elements of Dunhuang caisson pattern and applied them in fashion design

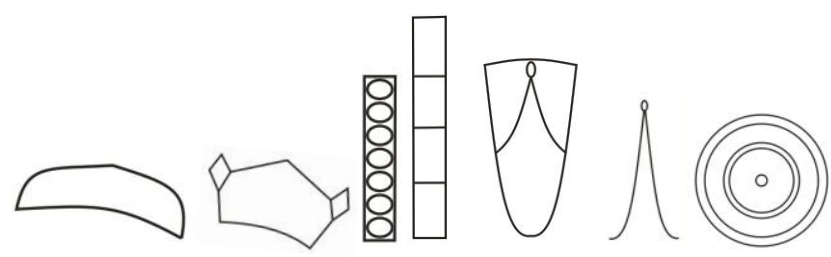

Figure 15. Elements decomposition map of caisson pattern 
After decomposition patterns, it can be seen that simple geometric patterns always be used in the application of multi-caisson, based the constitution of point, line, surface, the same point also used in the modern aesthetic principles. Now, composed the simple elements of Figure 15 to a new constitution, attempted to get different patterns, and with different colors applied to fashion design, explore different results.

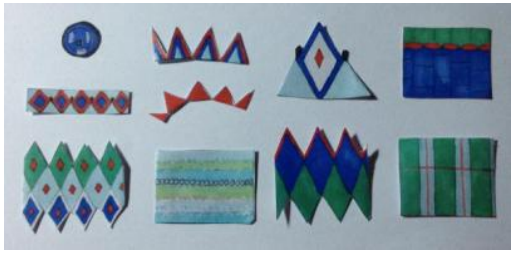

Figure 16. Regroup the graphics of constituent elements

Regroup the elements of Figure 16, mainly in diamond pattern, composed rectangular and round. Drawing tools are markers and color of lead, the main use of color painted wood in Montgomery 01 red, 20 ultramarine, 48 light green. On the basis of the constituent elements of Figure 16, ordering the elements. Based on a rectangular horizontal arrangement at the upper half of clothing, mainly use constituent elements in the waist and below the waist, arranged in a rectangle on the vertical drawing interspersed to do in diamond design, vertical arrangement reflects the tension in the visual effects. Showing the romantic atmosphere of bohemian style at modern times.

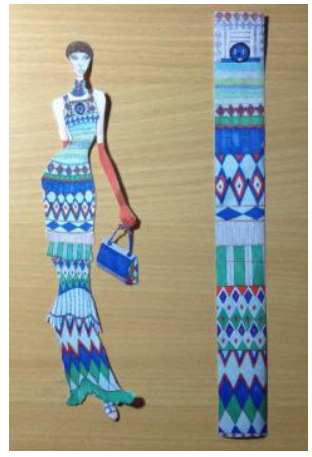

Figure 17. Pattern sketch of dress (b)

\subsection{Application of inherent color in Dunhuang caisson pattern}

Inherent color of Caisson pattern correspond to modern color mainly based on US PANTONE Color Card ( 2726 164 ), produce harmony between warm and cold colors hue, use its inherent color, with casual and comfortable design make fashion design. Urban casual style brings a sense of ease, shows the personality and pleasure at the same time, the designing of the clothes is mainly simple, without too much decoration, but with a certain degree of fashion. Extracted color from the caisson chart, note the depth of change in the use to make clothing in the three-dimensional visual effect.

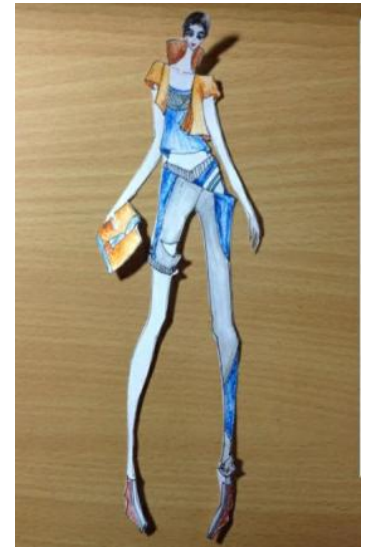

Figure 18. Pattern sketch of dress (c)

\section{CONCLUSION}

Based on the analysis of Dunhuang caisson pattern, combined with the present situation of research status, analyze the current research methods of caisson pattern in modern design, and summarize its findings. Select the appropriate method to use in fashion design, from the application of the extracted elements to application of reorganized elements and finally to the application of color, combined with modern clothing fashion style and popular style, designed three different styles of clothing. Combined the Chinese traditional elements and modern design to make my own clothing designs, retaining local feature of the original patterns, but with a certain sense of social fashion. This exploration of traditional culture and patterns with distinctive ethnic characteristics is a good research, it is worth going in-depth analysis.

\section{ACKNOWLEDGEMENTS}

The National Natural Science Foundation of China (No. 51105145), Project on the Integration of Industry, Education and Research of Guangdong Province(2012B091100446), Open Project of Key Laboratory of Innovation Method and Decision Management System of Guangdong Province (2011A060901001-05C)

\section{REFERENCES}

[1] Chen Xiongjun, Liang Zhaohua "numerous in simplified Analysis of Dunhuang caisson pattern" Xi'an Institute of Technology Journal Vol 18 March 2004 the first phase.

[2] Liu Tao. Peng Maifu "Dunhuang caisson pattern in modern interior design application." Beijing University of Science and Disaster Prevention. Internet Retrieval: http://www.artdesign.org.cn/

[3] Xiao Xiao "from Dunhuang caisson pattern dyeing patterns of Modern Design" theory modern decor in September 2012. 